Check for updates

Cite this: Mater. Adv., 2020, 1,1364

Received 2nd April 2020, Accepted 9th July 2020

DOI: $10.1039 / \mathrm{d} 0 \mathrm{ma00159g}$

rsc.li/materials-advances

\title{
Concentrated aqueous dispersions of low-defect few-layer thick graphene using surface active ionic liquid for enhanced enzyme activity $\dagger$
}

\author{
Gagandeep Singh, $\ddagger^{a}$ Manvir Kaur, $\stackrel{\ddagger}{ }^{a}$ Gurbir Singh, ${ }^{\text {ab }}$ Komal Arora, ${ }^{a}$ Manpreet Singh, ${ }^{a}$ \\ Bilal A. Sheikh (iD ${ }^{a}$ and Tejwant S. Kang (D) *a
}

\begin{abstract}
Low energy ultrasonic assisted aqueous phase exfoliation of defect free graphene ( $D / G=0.19)$ in a high yield $\left(3.0 \mathrm{mg} \mathrm{ml}^{-1}\right.$ ) is achieved using benzimidazolium based surface active ionic liquid (SAIL) appended with an ester functionalized alkyl chain. The obtained dispersions are found to be stable against agglomeration or sedimentation for at least 15 days, owing to coulombic repulsions offered by electron deficient $\pi$-conjugated SAIL ions adsorbed onto graphene. The exfoliated few-layer thick graphene sheets (2 layers $53 \%$ and 3-5 layers $20 \%$ ) offer a high surface area in conjunction with high concentration, which is exploited for the efficient loading of an enzyme, cellulase, via hydrophobic interactions. The graphene appended cellulase is found to exhibit a remarkable increase in the enzyme activity (13-fold) as compared to cellulase in the buffer. It is expected that the present results would act as a platform for the synthesis and utilization of new SAILs for graphene exfoliation, stabilization, and functionalization required to widen the application arena of graphene.
\end{abstract}

\section{Introduction}

Since its introduction, graphene, ${ }^{1}$ owing to its unique physicochemical properties, ${ }^{2-8}$ has attracted great interest for a wide array of applications. ${ }^{9-11}$ One such application is the use of graphene as a support for enzyme loading owing to its large surface area. In past, different enzymes such as lipase, ${ }^{12,13}$ horseradish peroxidase, ${ }^{14,15}$ and cellulase ${ }^{16,17}$ have been successfully immobilized onto graphene oxide (GO) via electrostatic $\mathrm{c}^{12,15,16}$ or covalent interactions ${ }^{13,14}$ due to the presence of functional groups on GO; however, graphene has not been investigated for the same purpose. The immobilization of enzymes onto GO has been marked by improved thermal ${ }^{13,15}$ and solvent stability of enzyme along with reusability. ${ }^{16}$ Such immobilized enzymes retains enzymatic activity, however, a decrease in the enzyme activity has been reported as compared to the bare enzyme, particularly in the case of enzymes adsorbed onto GO via a covalent linkage. ${ }^{18}$ Alterations in the secondary $\left(2^{\circ}\right)$ or

\footnotetext{
${ }^{a}$ Department of Chemistry, University Grants Commission (UGC) Centre for Advanced Studies-II, Guru Nanak Dev University, Amritsar-143005, Punjab, India. E-mail: tejwant.chem@gndu.ac.in, tejwantsinghkang@gmail.com; Tel: +91-183-2258802 ext. 3291

${ }^{b}$ Department of Chemistry and Biochemistry, Graduate School of Engineering, Kyushu University, 744 Motooka, Nishi-ku, Fukuoka 819-0395, Japan

$\dagger$ Electronic supplementary information (ESI) available. See DOI: 10.1039/ d0ma00159g

\# G. S. and M. K. contributed equally to this research work.
}

tertiary $\left(3^{\circ}\right)$ structure of enzymes and hence the deformation of the active site of an enzyme at the surface of GO could be a reason for decreased enzymatic activity. ${ }^{14}$ On the other hand, the enzyme adsorbed physically on GO has been reported to exhibit higher activity as compared to that tethered covalently. ${ }^{14}$

It is conceived that defect-free graphene instead of GO would offer a more suitable surface with the high surface area for enzyme loading via physical-adsorption exploiting hydrophobic interactions. Such weaker interactions are not expected to alter the microenvironment of the active site of an enzyme. Further, the aqueous solutions of well-dispersed single-layered or a few-layered thick graphene would result in a higher surface area required for efficient enzyme loading. The high stability of graphene dispersion against agglomeration and phase separation is another critical aspect to achieve the best enzyme activity as agglomeration and phase separation of enzyme-loaded graphene would decrease the concentration of enzyme dispersed in an aqueous medium, hence decreasing the enzyme activity. Therefore, new eco-friendly and cost-effective methods for the exfoliation of very thin defect-free graphene in an aqueous medium with high dispersion stability and subsequent enzyme loading, where enzymes show enhanced activities, should be devised to enhance the application arena of graphene.

In past, different methods $s^{1,19-23}$ have been employed for the preparation of graphene from graphite; however, these methods have certain limitations. The ultrasonic-assisted exfoliation of graphene in organic solvents of appropriate surface energy is 
another route that produces high quality graphene, ${ }^{24-29}$ but this process is limited by the high toxicity of organic solvents. Ionic liquids (ILs), an emerging class of green solvents, have also been used for graphene exfoliation ${ }^{30,31}$ but they confront the problem of high cost when used neat. Therefore, the best solvent for the liquid phase exfoliation of graphene is water, where the surface energy is lowered by the addition of surfactants that not only helps in exfoliation but also assists in the long-term stabilization of the colloidal solutions of graphene against agglomeration. ${ }^{32-39}$ Although this method seems to be most appropriate for the mass production of graphene, a low concentration $\left(<0.5 \mathrm{mg} \mathrm{ml}^{-1}\right)$ of exfoliated graphene in aqueousconventional surfactant solutions is the major drawback.

Herein, we report the direct exfoliation of graphene in an aqueous medium up to high concentrations $\left(3.0 \mathrm{mg} \mathrm{ml}^{-1}\right)$, which exhibits long-term stability towards agglomeration, using ester-functionalized benzimidazolium based surface active IL (SAIL) (Scheme 1).

The better surface active properties and designer nature of SAILs $^{40-48}$ over conventional ionic surfactants prompted us to evaluate the graphene exfoliation efficiency and stability of the colloidal dispersions of graphene in aqueous SAILs. The ease of the synthetic procedure ${ }^{40}$ of SAIL would be beneficial in scaling-up the process required for the mass-production of graphene in a cost-effective way. The employed SAIL also exhibits a low critical aggregation concentration $(\mathrm{cac})^{40}$ of $\approx 1.4 \mathrm{mmol} \mathrm{L}^{-1}$ in an aqueous medium, which also would add to the economy of the exfoliation process as the maximum exfoliation of graphene has been found to be achieved around cac of conventional ionic surfactants. ${ }^{33}$ The choice of the benzimidazolium cationic head group in SAIL is governed by its planner and $\pi$-conjugated electron deficient nature, which would lead to the efficient adsorption of SAIL onto graphene surface via $\pi-\pi$ interactions supplemented by hydrophobic interactions, required for the desirable exfoliation of graphene. Further, $H$-bonding prone ester-moiety is expected to anchor with graphene sheets while retaining the hydrating water, which not only would help in exfoliation but would also add to the colloidal stability. In this way, the present study represents a new and efficient low energy ultrasonic-assisted method for the mass production of highly concentrated, relatively defect-free few-layered graphene in an aqueous medium exhibiting desired colloidal stability for different applications. The exfoliated graphene is utilized as a support for the loading

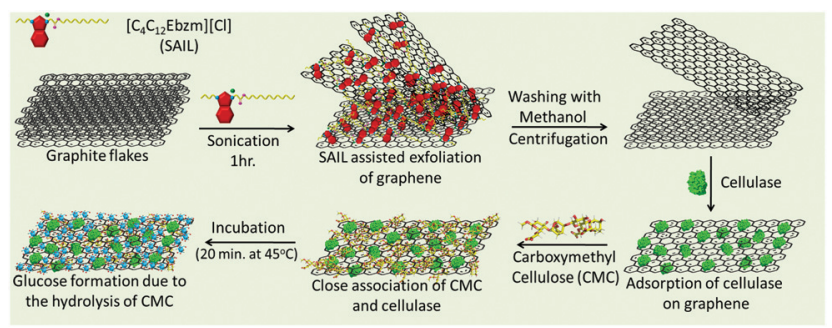

Scheme 1 SAIL-assisted exfoliation of graphene and loading of cellulase on exfoliated graphene. of cellulase, where a 13-fold increase in an enzyme activity is observed as compared to the bare enzyme. At first, we shall discuss the efficiency of the exfoliation process and the quality of exfoliated graphene, followed by its utilization as support for cellulase loading and role in enhanced enzyme activity.

\section{Result and discussion}

The detailed aspects of graphene exfoliation and characterization are provided in the Experimental section (Annexure S1, $\mathrm{ESI} \dagger$ ). The absorption spectra of graphene (Fig. 1A) dispersion were measured and found to be flat and featureless as expected for quasi-2D materials. ${ }^{49}$ The absorption coefficient, $\alpha$, which is an indicator of dispersion stability, is found to be $747 \mathrm{ml} \mathrm{mg}^{-1} \mathrm{~m}^{-1}$, which is in good agreement with that reported previously. ${ }^{50}$ The concentration of exfoliated graphene in aqueous supernatant was calculated by the careful measurement of the filtered mass of graphene, after drying, accounting for the mass of residual SAIL. The amount of exfoliated graphene was found to increase with the increase in the concentration of SAIL up to cac, which decreases thereafter (Fig. 1B). At concentrations much lower or higher than $c a c$, the dispersions were found to be phase-separated just after 24 hours (Fig. S1, ESI $\dagger$ ). As shown in Fig. 1C, at optimum $[\mathrm{SAIL}]=1.0 \mathrm{mM}$, a graphene dispersion of $3.0 \mathrm{mg} \mathrm{ml}^{-1}$ is obtained. It is important to mention that the cac of SAIL is found to be shifted from $1.4 \mathrm{mM}$ to $1.0 \mathrm{mM}$ in the presence of graphene. During the exfoliation of graphene in an aqueous medium, the hydrophobic surface of graphene sheets are in great demand for the adsorption of SAIL moieties. Relatively higher concentration of SAIL on the graphene surface as compared
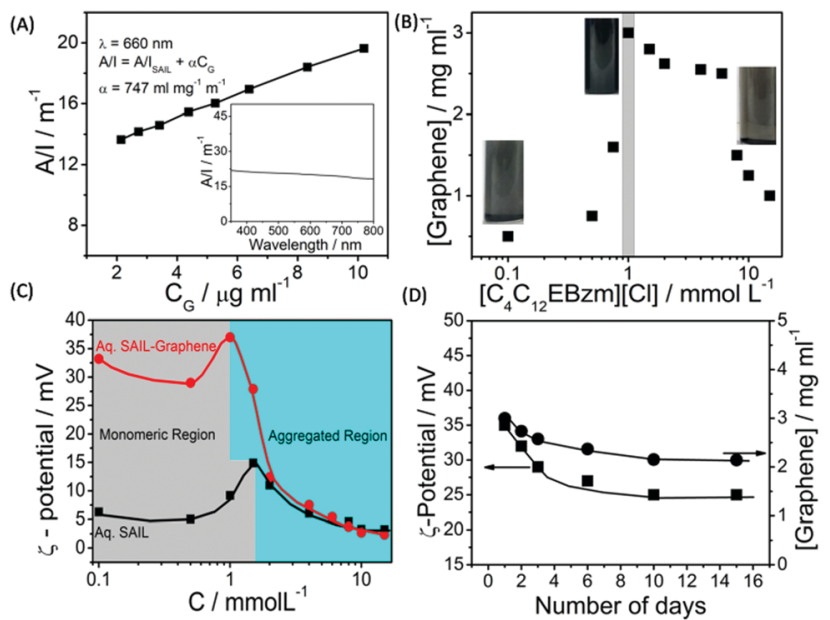

Fig. 1 Absorbance per unit length at $660 \mathrm{~nm}$ as a function of graphene concentration $\left(\mathrm{C}_{\mathrm{G}}\right)$ (Inset shows the absorbance spectra of graphene dispersion) (A); effect of the concentration of SAIL on the extent of graphene exfoliation (B); the variation of $\zeta$-potential in aqueous SAIL solutions in the absence and presence of graphene (C) and the variation of $\zeta$-potential and residual content of exfoliated graphene in aqueous dispersions as a function of time (D) at $298.15 \mathrm{~K}$. As per the law of mass action, the number of surfactant monomers below cac in a solution is equal to cac $^{52}$ the increase in the number of which increases the extent of penetration in graphene layers and hence greater exfoliation is observed. 
to that present in bulk resulted in the advancement of the selfassembly of SAIL similar to that reported in literature. ${ }^{51}$

The obtained concentration of graphene is much higher than that reported for conventional ionic surfactants (0.1-0.5 mg ml$\left.{ }^{-1}\right) .{ }^{33-39}$ The content of exfoliated graphene is higher than that obtained using large ionic aromatic molecules $\left(>0.1 \mathrm{mg} \mathrm{ml}^{-1}\right)^{53-57}$ and then that obtained $\left(0.1-1 \mathrm{mg} \mathrm{ml}^{-1}\right)$ for the exfoliation of GO expanded by the intercalation of $\mathrm{H}_{2} \mathrm{SO}_{4} / \mathrm{HNO}_{3}{ }^{58}$ It has been shown that surfactants having ionic groups attached to an electron deficient $\pi$-conjugated unit through alkyl spacers exhibit high tendency to exfoliate (1.2-5.0 $\mathrm{mg} \mathrm{ml}^{-1}$ ) graphene depending on the centrifugation rate. ${ }^{59}$ The used SAIL is highly efficient in promoting the exfoliation of graphene without oxidation in high concentrations in an economical manner. This is attributable to the presence of $\pi-\pi$ interactions between graphene and aromatic benzimidazolium ring supplemented by the hydrophobic and electrostatic forces of interaction with graphene.

Further, the similarity in concentration of SAIL at which maximum graphene exfoliation is observed to that where the maximum magnitude of $\zeta$-potential is observed (Fig. 1C) confirms the electrostatic repulsion between adsorbed SAILs onto graphene as the driving force behind colloidal stability. Owing to the flexible nature provided by the ester moiety, ${ }^{44}$ SAIL could adopt varying orientations for an optimal contact with graphene, resulting in efficient exfoliation. Further, the ester-group is expected to retain hydrating water molecules along with SAIL via $H$-bonding to provide colloidal stability to exfoliated graphene.

Thus, formed graphene dispersions after centrifugation $\left([\mathrm{SAIL}]=1.0 \mathrm{mM},\left[C_{\mathrm{G}}\right]=3.0 \mathrm{mg} \mathrm{ml}^{-1}\right)$ were tested for stability against agglomeration and sedimentation. The formed grapheneSAIL dispersions were found to be stable for at least 15 days against agglomeration as suggested by the negligible change in the concentration of graphene in dispersion (Fig. 1D). However, initially, the content of dispersed graphene decreases by $\approx 17 \%$ from $3.0 \mathrm{mg} \mathrm{ml}^{-1}$ to $2.5 \mathrm{mg} \mathrm{ml}^{-1}$ for 2 days. Further, $\zeta$-potential, which is an important parameter to monitor the colloidal stability, is found to be $+35 \mathrm{mV}$, which is much above than the colloidal stability limit of $+25 \mathrm{mV}$. It is to be noted that the $\zeta$-potential of the graphene dispersion is found to be more than twice of that observed in the absence of graphene at the SAIL concentration where maximum exfoliation takes place (Fig. 1D). This shows the efficient adsorption of SAIL ions on to the graphene surface, required for efficient exfoliation and colloidal stability (Scheme 1).

The exfoliated graphene was characterized via powder X-ray diffraction, Raman spectroscopy, transmission electron microscopy (TEM), and atomic force microscopy (AFM) techniques. An efficient exfoliation of graphene is suggested via XRD measurements, where in the case of exfoliated graphene only a broad band is observed as compared to a sharp diffraction peak at $26.5^{\circ}$ in the case of graphite (Fig. 2A). Fig. 2B shows the Raman spectra obtained for exfoliated graphene sheets. The presence of $\mathrm{G}\left(1580 \mathrm{~cm}^{-1}\right)$ and $\mathrm{D}\left(1350 \mathrm{~cm}^{-1}\right)$ bands is assigned to the $\mathrm{sp}^{2}$-hybridized nature of carbon of graphene. The appearance of a relatively stronger D-band in graphene as compared to
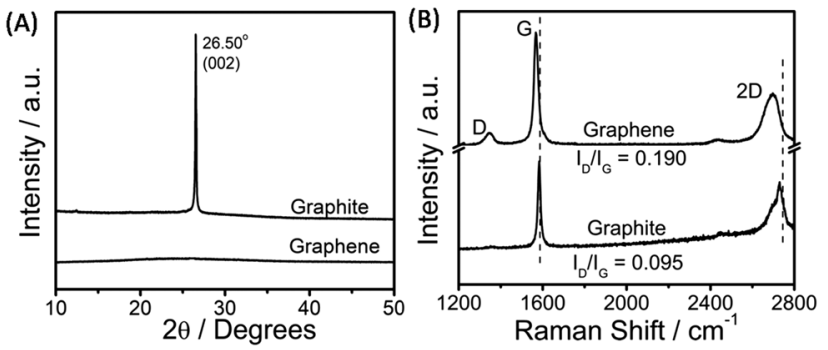

Fig. 2 X-ray diffraction pattern (A) and the obtained Raman spectra (B) for pristine graphite and exfoliated graphene.

that of graphite indicates the presence of edge or basal defects; however, a lower intensity ratio of $\mathrm{D} / \mathrm{G}$ bands $(\mathrm{D} / \mathrm{G}=0.190)$ suggests the smaller extent of these defects ${ }^{33,60}$ as compared to that observed in the presence of sodium cholate $(\mathrm{D} / \mathrm{G}=0.57$, on average). ${ }^{34}$ The shift and shape of the $2 \mathrm{D}$ band of graphene is peculiar of a few-layer thick ( $<5$ layers) graphene, ${ }^{33,59}$ while the shift in the G-band attributes to the change in the electronic structure of graphene due to the $\pi-\pi$ interactions between graphene and aromatic benzimidazolium ring.

Fig. 3(A-D) shows the TEM images of exfoliated graphene flakes of different thickness along with selected area electron diffraction (SAED) pattern. High resolution TEM image (Inset Fig. 3A and C) of very thin graphene flakes suggest the presence of 3-4 layered graphene. The observed SAED (Fig. 3D) pattern is peculiar of the hexagonally arranged lattice of graphene, where the higher intensity of the second diffraction layer as compared to the first one suggests the exfoliation of two/three layered thick graphene. ${ }^{60}$

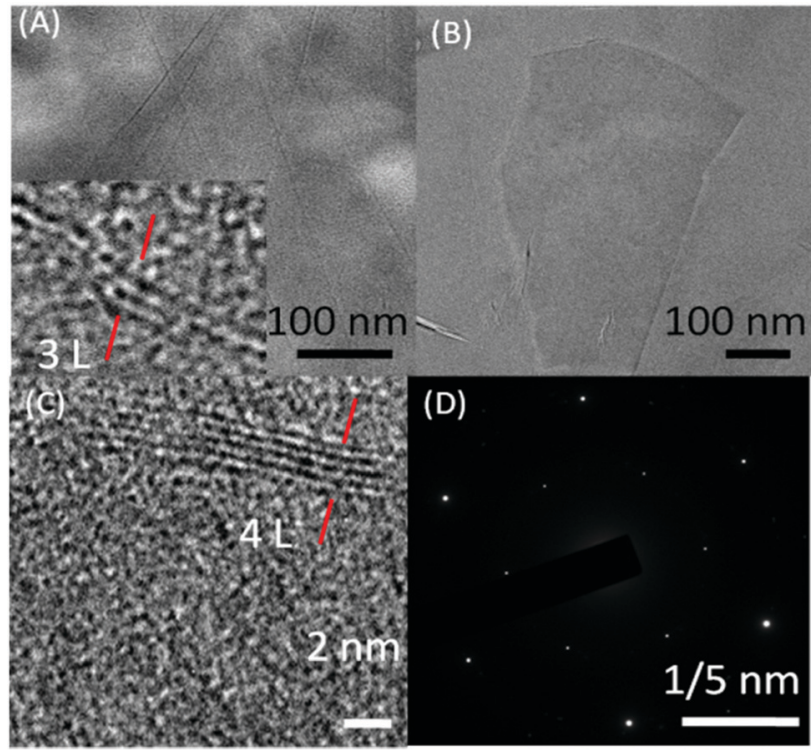

Fig. 3 TEM images of exfoliated graphene as long flakes $(A)$; smaller but few-layer thick flake (B); high resolution TEM images near the edge of a few-layer graphene flake showing the number of graphene layers (C); and SAED pattern of exfoliated graphene flakes (D). The inset of (A) shows the number of graphene layers as observed at the edge of an exfoliated graphene flake. 
(A)
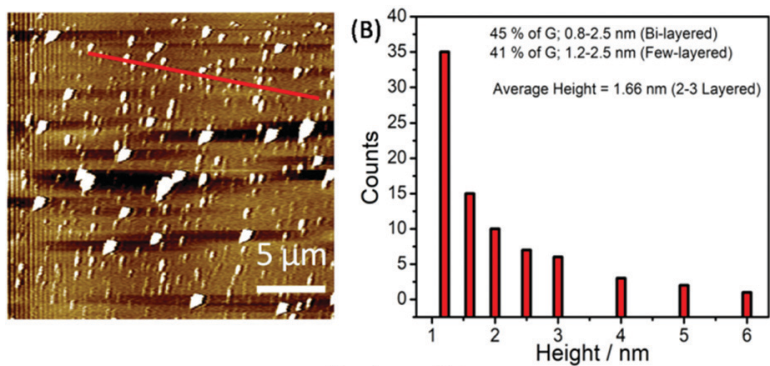

(C) 2.0

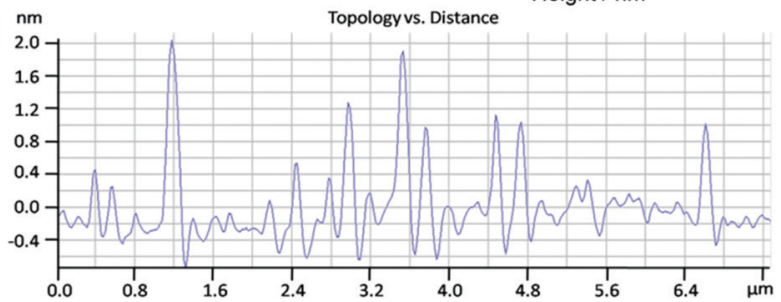

Fig. 4 AFM image of the exfoliated graphene flakes (A); the statistical analysis of the obtained height profile (B) and surface topology vs. the distance profile of exfoliated graphene flakes (C) corresponding to the red line shown in $(A)$.

To statistically analyze the size distribution of graphene flakes, AFM measurements (Fig. 4A-C) have been carried out after depositing the graphene on mica sheets. Graphene sheets with a lateral size of 50-500 $\mathrm{nm}$ have been obtained with a mean lateral size of $\approx 150 \mathrm{~nm}$. The analysis of AFM images (Fig. 4B and C) suggests that $\approx 45 \%$ of the flakes comprised two layers, exhibiting a thickness of $0.8-1.2 \mathrm{~nm} .42 \%$ of the analyzed flakes are found to be few-layered thick with a thickness of 1.2-3.0 nm.

To probe the mechanism of the SAIL-mediated exfoliation of graphene, high disperse-ability and colloidal stability, steady-state fluorescence, $2 \mathrm{D}{ }^{1} \mathrm{H}-{ }^{1} \mathrm{H}$ NMR measurements, and computational investigations were performed. A decrease in the intensity of inherent fluorescence exhibited by SAIL ${ }^{40}$ with an increase in the content of dispersed graphene is observed (Fig. S2, ESI $\dagger$ ). This is attributed to the interactions of the $\pi$-conjugated electron-deficient benzimidazolium head group of SAIL aided by its planer nature with the electron-rich and planner surface of graphene. The appearance of $2 \mathrm{D}{ }^{1} \mathrm{H}-{ }^{1} \mathrm{H}$ NOESY cross-peaks between protons of the cationic head group and hydrophobic alkyl chain of SAIL in the presence of graphene, which otherwise are absent suggests their adsorption at the graphene surface (Fig. S3, ESI $\dagger$ ). The calculated absorption free energy of SAIL onto the surface of graphene comes out to be $-13.76 \mathrm{kcal} \mathrm{mol}^{-1}$, which comes out to be close to that observed for the naphthalene diimide-based ionic surfactant having a carboxylate ionic head group and more than that of conventional ionic surfactants. ${ }^{59}$ This suggests a high affinity of SAIL towards the graphene surface. Both the reported surfactants $^{59}$ and SAIL used in this work have the $\pi$-conjugated electron-deficient system; however, the extent of conjugation in case of SAIL is less, even then, an appreciable absorption energy has been observed. This is attributable to the presence of the relatively flexible ester moiety near the ionic head group by virtue of which the ionic head group could adopt different orientations
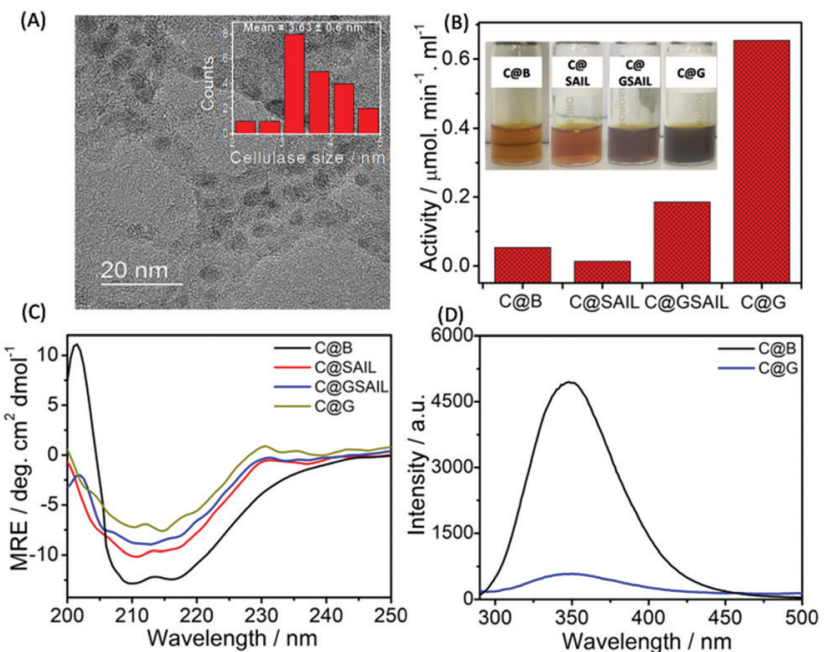

Fig. 5 TEM image of C (aG (A); activity of cellulase towards CMC adsorbed on washed graphene, on graphene with SAIL, in the buffer and in the SAIL aqueous medium (B); far UV CD spectra of native cellulase, cellulase adsorbed on graphene, on graphene with SAIL and in the SAIL aqueous medium (C); and intrinsic fluorescence of native cellulase and cellulase adsorbed on washed graphene (D). (Inset of A shows the histograms showing the mean particle size of cellulase along with standard deviation.)

for efficient absorption at the graphene surface. The retention of $H$-bonded water with the $H$-bonding prone ester moiety is expected to retard agglomeration.

Further, SAIL was removed from exfoliated graphene via the method mentioned in Annexure S1, ESI, $\dagger$ and the removal of SAIL has been confirmed via the thermogravimetric analysis (Fig. S4, ESI $\dagger$ ). Thus, obtained graphene is used as nano-support for the loading of cellulase, and enzyme activity of the loaded cellulase is investigated. Cellulase has been immobilized on graphene using a cellulase buffer solution at $\mathrm{pH}$ 4.8. The TEM image shown in Fig. 5A suggests the adsorption of cellulase on graphene, which is also supported via AFM measurements (Fig. S5 and S6, ESI $\dagger$ ). The presence of lattice planes having a distance of $0.261 \mathrm{~nm}$ indicates the crystalline nature of cellulase adsorbed on graphene with an interplanar distance of $0.34 \mathrm{~nm}$ (Fig. S7A, ESI $\dagger$ ).

The functional stability of cellulase adsorbed onto the surface of graphene (C@G) is probed by measuring its enzyme activity towards carboxymethyl cellulose (CMC) (Annexure S1 and S2, ESI $\dagger$ ) and is compared with that observed in the case of cellulase dispersed in the buffer (C@B), in aqueous SAIL solution (C@SAIL) and in the aqueous dispersions of SAIL and graphene (C@GSAIL) (Fig. 5B). The enzyme activity is found to be remarkably enhanced in the case of C@G (13-fold), whereas it enhances appreciably in the case of C@GSAIL (3.4-fold) as compared to that observed in the case of C@B. On the other hand, a decrease in the enzyme activity is observed in the case of G@SAIL.

A 13-fold increase in the enzyme activity in the case of C@G is assigned to favorable conformational changes in secondary $\left(2^{\circ}\right)$ structure of cellulase, which opens up the active sites of enzyme resulting in enhanced enzyme activity. The conformational changes in cellulase in the form of C@G are confirmed 
from far-UV CD measurements (Fig. 5C). The $2^{\circ}$-structure of cellulase mainly comprises $\alpha$-helices, $\beta$-sheets and, turns, and the bands present at the $-\theta_{209}$ and $-\theta_{217}$ corresponds to the presence of $\alpha$-helices and $\beta$-sheets, respectively ${ }^{61}$ The alterations in the $2^{\circ}$-structure of cellulase in the case of C@G are evidenced by a decrease in the magnitude of MRE values corresponding to $-\theta_{209}$ and $-\theta_{217}$ along with the change in the shape of $\mathrm{CD}$ spectra owing to the unfolding of cellulase. Further, a drastic decrease in the inherent fluorescence intensity of cellulase, which mainly arises from 7 Tryptophan residues present at various sites of enzyme, ${ }^{62}$ is observed when cellulase is appended with graphene (Fig. 5D). This indicates the destabilization of the $3^{\circ}$-structure of cellulase owing to hydrophobic interactions with graphene in the case of C@G, which exposes the Trp residues towards a relatively more polar environment. Two each of the Trp residues are present in the active site tunnel of cellulase and near the catalytic site, which provides hydrophobic stacking interactions for glycosyl units and are essential for substrate loading. ${ }^{63}$ Therefore, it is natural to assume that the unfolding of cellulase modifies the active as well as the catalytic site of an enzyme for efficient substrate binding and enzymatic activity. The fluorescence bands of cellulase and SAIL overlap with each other in the case of C@SAIL and C@GSAIL (Fig. S8, ESI †). Therefore, it was difficult to discuss the structural stability of cellulase in presence of SAIL using fluorescence measurements. The relatively lesser increase in the enzyme activity in the case of C@GSAIL is assigned to the presence of SAIL. Similar to earlier reported SAIL-protein systems, SAIL is expected to interact not only with cellulase but also with the substrate $(\mathrm{CMC})^{64}$ via electrostatic and hydrophobic interactions. The interactions of SAIL in C@SAIL with cellulase results in structuring rather than unfolding of cellulase marked by an increase in the magnitude of MRE values, corresponding to $-\theta_{209}$ and $-\theta_{217}$ with the decreased content of turns. This results in the decreased enzyme activity as compared to that observed in buffer (Fig. 5B). In the case of C@GSAIL, there work two forces, one is the hydrophobic interactions of cellulase with the graphene surface, which favors increased enzyme activity and the other is structuring of the enzyme via interactions with SAIL, which disfavors the enzyme activity. The dominance of the graphene effect in the case of C@GSAIL results in increased enzyme activity but to a relatively lesser extent as compared to C@G.

Further, the colloidal stability of the C@GSAIL dispersion has been found to be at least 15 days due to the presence of electrostatic repulsions between SAIL ions adsorbed on graphene. However, colloidal stability reduces to three days in the absence of SAIL (C@G). The observed stability of the C@G dispersion could be assigned to the presence of highly hydrated cellulase at the surface of graphene. Even though C@G dispersion possesses a lower colloidal stability, the enzymatic activity of cellulase in C@G is retained, up to $41 \%$ of its original activity, after a period of 15 days (Fig. S9, ESI $\dagger$ ). It is important to mention that the enzyme activity decreases both in C@G as well as in C@B with time. The observed activity is still higher than that observed for C@B. Hence, the prepared C@G would lay a new platform to establish systems with enhanced enzymatic activity. In this way, a new approach for the efficient exfoliation of defect-free graphene in high concentration for achieving remarkably enhanced enzyme activity is developed.

\section{Conclusions}

Surface active ionic liquid (SAIL) having a positively charged benzimidazolium based aromatic head group appended with an ester-functionalized alkyl chain is used to achieve highly stable and concentrated aqueous dispersion of few-layer thick graphene. In comparison to conventional surfactants, the exfoliation of graphene with the used SAIL is better even at a very low concentration of SAIL. Further, the formed defectfree graphene is used as an enzyme-immobilizing substrate for the adsorption of cellulase that exhibits 13-fold increase in the catalytic activity as compared to native enzyme in buffer. This is assigned to the structuring of the active site of enzyme when adsorbed physically onto the surface of graphene. It is anticipated that the content of graphene, colloidal stability and exclusive monolayer formation may be achieved by a decisive choice of cation, anion and functionalization over the alkyl chain of SAILs. Therefore, the present study along with previous reports on the use of ILs ${ }^{65,66}$ and surfactants ${ }^{32-36}$ for graphene exfoliation would offer a new platform for exploiting SAILs in an aqueous medium in conjunction with other non-conventional methods such as hydrothermal method ${ }^{67}$ for the efficient exfoliation and stabilization of a defect-free single-layer graphene in an aqueous medium.

\section{Conflicts of interest}

There are no conflicts to declare.

\section{Acknowledgements}

This work is supported by DST, Govt. of India wide project number SB/FT/CS-057/2013. Gurbir, Manvir, Komal and Manpreet are thankful to CSIR and UGC, Govt. of India, respectively for Fellowship. The infrastructure facility provided under the UPE, PURSE and CAS grant for this work is highly acknowledged.

\section{Notes and references}

1 K. S. Novoselov, A. K. Geim, S. V. Morozov, D. Jiang, Y. Zhang, S. V. Dubonos, I. V. Grigorieva and A. A. Firsov, Science, 2004, 306, 666.

2 K. S. Novoselov, A. K. Geim, S. V. Morozov, D. Jiang, M. I. Katsnelson, I. V. Grigorieva, S. V. Dubonos and A. A. Firsov, Nature, 2005, 438, 197.

3 Y. Zhang, Y. W. Tan, H. L. Stormer and P. Kim, Nature, 2005, 438, 201.

4 A. K. Geim and K. S. Novoselov, Nat. Mater., 2007, 6, 183.

5 Y. Wang, Y. Huang, Y. Song, X. Zhang, Y. Ma, J. Liang and Y. Chen, Nano Lett., 2009, 9, 220. 
6 D. Li and R. B. Kaner, Science, 2008, 320, 1170.

7 C. Lee, X. Wei, J. W. Kysar and J. Hone, Science, 2008, 321, 385.

8 A. A. Balandin, S. Ghosh, W. Bao, I. Calizo, D. Teweldebrhan, F. Miao and C. N. Lau, Nano Lett., 2008, 8, 902.

9 C. X. Guo, H. B. Yang, Z. M. Sheng, Z. S. Lu, Q. L. Song and C. M. Li, Angew. Chem. Int. Ed., 2010, 49, 3014.

10 M. J. Allen, V. C. Tung and R. B. Kaner, Chem. Rev., 2010, 110, 132.

11 L. Dai, Acc. Chem. Res., 2013, 46, 31.

12 W. Xu, Z. Fu, G. Chen, Z. Wang, Y. Jian, Y. Zhang, G. Jiang, D. Lu, J. Wu and Z. Liu, Nat. Commun., 2019, 10, 2684.

13 S. Hermanová, M. Zarevúcká, D. Bouša, M. Pumera and Z. Sofěr, Nanoscale, 2015, 7, 5852.

14 Y. Zhang, J. Zhang, X. Huang, X. Zhou, H. Wu and S. Guo, Small, 2012, 8, 154.

15 F. Zhang, B. Zheng, J. Zhang, X. Huang, H. Liu, S. Guo and J. Zhang, J. Phys. Chem. C, 2010, 114, 8469.

16 A. A. Gokhale, J. Lu and I. Lee, J. Mol. Catal. B: Enzym., 2013, 90, 76.

17 N. Srivastava, M. Srivastava, V. K. Gupta, P. W. Ramteke and P. K. Mishra, Bioresour. Technol., 2018, 270, 337.

18 J. Xu, Z. Sheng, X. Wang, X. Liu, J. Xia, P. Xiong and B. He, Bioresour. Technol., 2016, 200, 1060.

19 S. Stankovich, R. D. Piner, X. Chen, N. Wu, S. T. Nguyen and R. S. Ruoff, J. Mater. Chem., 2006, 16, 155.

20 S. Stankovich, D. A. Dikin, R. D. Piner, K. A. Kohlhaas, A. Kleinhammes, Y. Jia, Y. Wu, S. T. Nguyen and R. S. Ruoff, Carbon, 2007, 45, 1558-1565.

21 S. Park and R. S. Ruoff, Nat. Nanotechnol., 2009, 4, 217.

22 W. Yang, G. Chen, Z. Shi, C. C. Liu, L. Zhang, G. Xie, M. Cheng, D. Wang, R. Yang, D. Shi, K. Watanabe, T. Taniguchi, Y. Yao, Y. Zhang and G. Zhang, Nat. Mater., 2013, 12, 792.

23 D. R. Dreyer, R. S. Ruoff and C. W. Bielawski, Angew. Chem. Int. Ed., 2010, 49, 9336.

24 Y. Hernandez, V. Nicolosi, M. Lotya, F. M. Blighe, Z. Sun, S. De, I. T. McGovern, B. Holland, M. Byrne, Y. K. Gun'ko, J. J. Boland, P. Niraj, G. Duesberg, S. Krishnamurthy, R. Goodhue, J. Hutchison, V. Scardaci, A. C. Ferrari and J. N. Coleman, Nat. Nanotechnol., 2008, 3, 563.

25 C. E. Hamilton, J. R. Lomeda, Z. Sun, J. M. Tour and A. R. Barron, Nano Lett., 2009, 9, 3460.

26 U. Khan, H. Porwal, A. O'Neill, K. Nawaz, P. May and J. N. Coleman, Langmuir, 2011, 27, 9077.

27 A. O'Neill, U. Khan, P. N. Nirmalraj, J. Boland and J. N. Coleman, J. Phys. Chem. C, 2011, 115, 5422.

28 A. B. Bourlinos, V. Georgakilas, R. Zboril, T. A. Steriotis and A. K. Stubos, Small, 2009, 5, 1841.

29 E. Y. Choi, W. S. Choi, Y. B. Lee and Y. Y. Noh, Nanotechnology, 2011, 22, 365601.

30 X. Wang, P. F. Fulvio, G. A. Baker, G. M. Veith, R. R. Unocic, S. M. Mahurin, M. Chi and S. Dai, Chem. Commun., 2010, 46, 4487.

31 D. Nuvoli, L. Valentini, V. Alzari, S. Scognamillo, S. B. Bon, M. Piccinini, J. Illescasd and A. Mariani, J. Mater. Chem., 2011, 21, 3428.
32 L. Guardia, M. J. Fernández-Merino, J. I. Paredes, P. SolísFernández, S. Villar-Rodil, A. Martínez-Alonso and J. M. D. Tascón, Carbon, 2011, 49, 1653.

33 M. Lotya, Y. Hernandez, P. J. King, R. J. Smith, V. Nicolosi, L. S. Karlsson, F. M. Blighe, S. De, Z. Wang, I. T. McGovern, G. S. Duesberg and J. N. Coleman, J. Am. Chem. Soc., 2009, 131, 3611.

34 M. Lotya, P. J. King, A. U. Khan, S. De and J. N. Coleman, ACS Nano, 2010, 4, 3155.

35 S. Vadukumpully, J. Paul and S. Valiyaveettil, Carbon, 2009, 47, 3288.

36 S. Lin, C. J. Shih, M. S. Strano and D. Blankschtein, J. Am. Chem. Soc., 2011, 133, 12810.

37 S. De, P. J. King, M. Lotya, A. O’Neill, E. M. Doherty, Y. Hernandez, G. S. Duesberg and J. N. Coleman, Small, 2010, 6, 458.

38 K. Zhang, L. Mao, L. L. Zhang, H. S. On Chan, X. S. Zhao and J. Wu, J. Mater. Chem., 2011, 21, 7302.

39 K. Nawaz, M. Ayub, M. B. Khan, A. Hussain, A. Q. Malik, M. B. K. Niazi, M. Hussain, A. U. Khan and N. Ul-Haq, Nanomater. Nanotechnol., 2016, 6, 14.

40 G. Singh, M. Kaur, M. Drechsler and T. S. Kang, Chem. Commun., 2018, 54, 2432.

41 H. Wang, L. Zhang, J. Wang, Z. Li and S. Zhang, Chem. Commun., 2013, 49, 5222.

42 S. Ghosh, C. Ghatak, C. Banerjee, S. Mandal, J. Kuchlyan and N. Sarkar, Langmuir, 2013, 29, 10066.

43 G. Singh, G. Singh and T. S. Kang, J. Phys. Chem. B, 2016, 120, 1092.

44 G. Singh, R. Kamboj, V. S. Mithu, V. Chauhan, T. Kaur, G. Kaur, S. Singh and T. S. Kang, J. Colloid Interface Sci., 2017, 496, 278.

45 M. Blesic, M. Swadzba-Kwasny, J. D. Holbrey, J. N. Canongia Lopes, K. R. Seddon and L. P. Rebelo, Phys. Chem. Chem. Phys., 2009, 11, 4260.

46 B. Dong, N. Li, L. Zheng, L. Yu and T. Inoue, Langmuir, 2007, 23, 4178.

47 S. Mandal, J. Kuchlyan, S. Ghosh, C. Banerjee, N. Kundu, D. Banik and N. Sarkar, J. Phys. Chem. B, 2014, 118, 5913.

48 H. Wang, J. Wang, S. Zhang and X. Xuan, J. Phys. Chem. B, 2008, 112, 16682.

49 D. S. L. Abergel and V. I. Fal'ko, Phys. Rev. B: Condens. Matter Mater. Phys., 2007, 75, 155430.

50 S. Wang, M. Yi and Z. Shen, RSC Adv., 2016, 6, 56705.

51 C. M. Wijmans and P. Linse, J. Phys. Chem., 1996, 100, 12583.

52 M. J. Rosen, Surfactants and Interfacial Phenomena, Wiley, New York, 2nd edn, 1989.

53 D. Parviz, S. Das, H. S. T. Ahmed, F. Irin, S. Bhattacharia and M. J. Green, ACS Nano, 2012, 6, 8857.

54 X. An, T. Simmons, R. Shah, C. Wolfe, K. M. Lewis, M. Washington, S. K. Nayak, S. Talapatra and S. Kar, Nano Lett., 2010, 10, 4295.

55 A. Ghosh, K. V. Rao, S. J. George and C. N. Rao, Chem. - Eur. J., 2010, 16, 2700.

56 D. W. Lee, T. Kim and M. Lee, Chem. Commun., 2011, 47, 8259. 
57 S. Sampath, A. N. Basuray, K. J. Hartlieb, T. Aytun, S. I. Stupp and J. F. Stoddart, Adv. Mater., 2013, 25, 2740.

58 V. Panwar, A. Chattree and K. Pal, Phys. E, 2015, 73, 235.

59 L. Zhang, Z. Zhang, C. He, L. Dai, J. Liu and L. Wang, ACS Nano, 2014, 8, 6663.

60 A. C. Ferrari, J. C. Meyer, V. Scardaci, C. Casiraghi, M. Lazzeri, F. Mauri, S. Piscanec, D. Jiang, K. S. Novoselov, S. Roth and A. K. Geim, Phys. Rev. Lett., 2006, 97, 187401.

61 P. Bharmoria, M. J. Mehta, I. Pancha and A. Kumar, J. Phys. Chem. B, 2014, 118, 9890.

62 M. B. Turner, S. K. Spear, J. G. Huddleston, J. D. Holbrey and R. D. Rogers, Green Chem., 2003, 5, 443.
63 A. Nakamura, T. Tsukada, S. Auer, T. Furuta, M. Wada, A. Koivula, K. Igarashi and M. Samejima, J. Biol. Chem., 2013, 288, 13503.

64 G. Singh, G. Singh and T. S. Kang, Phys. Chem. Chem. Phys., 2018, 20, 18528.

65 C. Wang, Y. Chen, K. Zhuo and J. Wang, Chem. Commun., 2013, 49, 3336.

66 M. Kaur, G. Singh, K. Damarla, G. Singh, H. Wang, J. Wang, V. K. Aswal, A. Kumar and T. S. Kang, Phys. Chem. Chem. Phys., 2019, 22, 169.

67 S. Yang, K. Zhuo, Z. Zhang, J. Liu, G. Bai and J. Wang, J. Phys. Chem. C, 2019, 123, 27969. 